\title{
Die „Anderen“ im Klassenzimmer: Othering im Kontext von DaZ in der Lehrer/innenbildung
}

\author{
Martina Turecek
}

\begin{abstract}
Zusammenfassung
Der folgende Beitrag ist dem Thema „Othering“ - im Sinne der Herstellung von sozial wirksamer Differenzierung zwischen Menschen „mit und ohne Migrationshintergrund" gewidmet und bezieht sich auf die schulische Situation in Österreich. Die Auseinandersetzung mit der Thematik erfolgt im Kontext eines Seminars im Rahmen der österreichischen Pflichtschullehrer/innenausbildung an der Pädagogischen Hochschule Wien. Zu Beginn des Beitrags werden wesentliche Fragestellungen der Migrationspädagogik erörtert: Anhand welcher Parameter erfolgt eine gesellschaftliche Grenzziehung zwischen Menschen mit und ohne Migrationsgeschichte? Wie wird diese gesellschaftliche Ordnung produziert und in welchen Bereichen leistet das Schulsystem Vorschub für die (Re)Produktion? Die Sensibilisierung für die Thematik soll (angehenden) Pädagog/innen helfen, angemessen und professionell im Schulalltag zu agieren. An die theoretische Auseinandersetzung schließt die dramapädagogische Didaktisierung eines literarischen Texts an. Anhand der autobiografischen Erzählung „In Between. Mein Leben in zwei Kulturen“ der jungen österreichischen Autorin Amina Mahdy soll die Dimension der gesellschaftlichen Praxis des „Othering“ ein Stück weit erlebbar gemacht werden. Der Originaltext ist im Anhang nachzulesen und wird durch ein kurzes Interview mit der Autorin ergänzt. Abschließende Bemerkungen zur didaktischen Aufbereitung des Themas „Othering“ im Rahmen des Seminars runden den Beitrag ab.
\end{abstract}

\section{Einleitung}

In meiner Wahlveranstaltung für Deutsch als Zweitsprache an der Pädagogischen Hochschule Wien für angehende Lehrkräfte im Pflichtschulbereich hat sich die Thematik des „Othering“ und die damit verbundene Diskussion um Bildungsbenachteiligung im österreichischen Schulsystem als wichtiger Teilaspekt herausgestellt. In diesem Beitrag möchte ich meine Vorgehensweise zur inhaltlichen Aufbereitung des Themas skizzieren, um Anregungen und Anknüpfungspunkte für Lehrende zur Verfügung zu stellen.

Einer kurzen Einführung in das Themengebiet folgt die dramapädagogische Bearbeitung des literarischen Kurztexts „In Between. Mein Leben in zwei 
Kulturen“ von Amina Mahdy, welche als Ausgangspunkt für Reflexion und Diskussion mit den Studierenden über Othering und (Alltags-)Rassismus dient. Ziel der Beschäftigung mit der Thematik im Seminar ist die Sensibilisierung der angehenden Lehrkräfte für gesellschaftliche Zuschreibungspraxen in Bezug auf Migration und Ethnizität sowie die Vermeidung von Etikettierungen und Fremdzuschreibungen, die zu Ausgrenzung führen. Damit verbunden ist die Selbstreflexion als Angehörige/r der „Mehrheitsgesellschaft" sowie als Lehrperson.

\section{2 „Menschen mit Migrationshintergrund“ - "Migrationsandere“"}

Die Bezeichnung „Menschen mit Migrationshintergrund“ hat - abseits statistischer Definitionen - bereits einen festen Platz im medialen und alltäglichen Sprachgebrauch. In den letzten Jahren wurde sie in Österreich und Deutschland mehrfach zum „Unwort des Jahres“ vorgeschlagen. Der häufig pejorativ gebrauchte Begriff ist äußerst unscharf in seiner Bezeichnung und fasst pauschalierend Menschen unter Einbezug der Merkmale Staatsangehörigkeit, Migrationsgeschichte (mehrerer Generationen), Aufenthaltsstatus und Ethnizität zu einer Gruppe zusammen. Zudem unterliegen die Sprachen der Menschen in der Migrationsgesellschaft einer sozialen Wertung durch jene, die sich als „Mehrheitsgesellschaft“ verstehen. Im schulischen Kontext wird die Bezeichnung „mit Migrationshintergrund“ in der Praxis zumeist in defizitär ausgerichteter Perspektive auf Kinder und Jugendliche aus bildungsfernen und sozial unterprivilegierten Schichten angewandt, die sich „sichtbar"von einer vagen Norm abheben, die durch deutsche Sprachkenntnisse, bildungssprachliche Kompetenzen, soziale und „kulturelle“ Unterschiede, Religionszugehörigkeit und/oder äußere Merkmale konstruiert wird.

Im migrationspädagogischen Kontext wird das Kunstwort „Migrationsandere“ verwendet, das nicht nur zum Ausdruck bringt, dass es in der (österreichischen) Migrationsgesellschaft vielfach die pauschalierende Zuschreibung zu den Personengruppen Migrant/innen und Nicht-Migrant/innen gibt, sondern dass diese Festschreibungen konstruiert sind. (Mecheril et al. 2010: 15-18) „Migrationsandere" stellen keine einheitliche Gruppe dar: Natio-ethno-kulturelle Unterschiede werden zur Beschreibung der Andersheit verwendet, um die Ordnung der Mehrheitsgesellschaft zu festigen.

\subsection{Schlüsselbegriff „Othering“}

Das Konzept des Othering stammt aus dem postkolonialen Diskurs und geht auf den Literaturwissenschaftler Edward Said zurück, der in den 1970er-Jahren damit eine Möglichkeit aufgezeigt hat, den Diskurs des Fremdmachens als eine gewaltvolle hegemoniale Praxis zu beschreiben. (Mecheril et al. 2010: 42) Othering ist ein weitverbreitetes Phänomen zur Herstellung von Asymmetrien 
in der Gesellschaft, die durch Unterscheidungen entstehen. Es kann als eine Hierarchisierung der Gesellschaft verstanden werden, wobei sich eine „Mehrheit" („Wir") von einer „Minderheit" („Nicht-Wir") durch Zuschreibungen an diese abgrenzt und sich als privilegiert darstellt. Eigene Rechte bzw. Vorteile können damit bestätigt werden, den minorisierten „Anderen“ wird der Zugang zu Ressourcen verweigert. Die durch Othering aufgestellten Hierarchien werden durch Prozesse der Diskriminierung und des Rassismus generiert. Diskriminierungen beruhen auf den Zuschreibungen verschiedener Merkmale wie Religion, Sprache, Nation, Ethnizität oder Kultur. (Mikura 2013: 8) Abgrenzung und Ausgrenzung dienen letztendlich der Herstellung und/oder Zementierung von gesellschaftlichen Machtverhältnissen. (Zick; Küpper, in Marschke; Brinkmann 2015: 95)

\subsection{Migrationsandere im österreichischen Schulsystem}

Welche Bedeutung besitzt die Praxis des Othering für die Schule bzw. für die Ausbildung von Lehrkräften? Wie lässt sich die Stellung von Schüler/innen mit Migrationsgeschichte im österreichischen Schulsystem beschreiben?

In Österreich verfügte 2012/2013 im Bundesdurchschnitt jede/r fünfte Schüler/in im Allgemeinen Pflichtschulbereich über eine andere Erstsprache als Deutsch, in Wien waren es mehr als 50\%. (BMBF 2014:24) Angesichts der Schülerpopulation wird deutlich, dass von einer „Mehrheitsgesellschaft“ gegenüber einer „Gruppe von Menschen mit Migrationshintergrund“ nicht gesprochen werden kann. Dennoch verhält sich das System Schule so, als stünde es einer monolingualen, monokulturellen Schülerschaft gegenüber. (Gogolin 2008) Mehrsprachigkeit und „kulturelle Andersheit“ werden vielfach als Probleme wahrgenommen, da sie der Normalitätskonstruktion des Bildungssystems nicht entsprechen.

In Bezug auf den Schulerfolg schneiden migrationsandere Schüler/innen in Österreich deutlich schlechter ab als Schüler/innen ohne Migrationsgeschichte. Das zeigen nicht nur internationale Bildungsstudien wie PISA, PIRLS und TIMSS, sondern diese Tatsache lässt sich auch an folgenden Faktoren ablesen: Schüler/innen mit Migrationsgeschichte wiederholen im Durchschnitt überproportional oft eine Klasse, sind an für höhere Schulabschlüsse qualifizierenden allgemeinbildenden Schulen unterrepräsentiert und an Sonderschulen sowie Neuen Mittelschulen überrepräsentiert. Dadurch ergeben sich Nachteile und Erschwernisse innerhalb der Schullaufbahn und längerfristig entsteht eine Benachteiligung in Hinblick auf die Ressourcen im gesellschaftlichen und beruflichen Leben. (Dirim et al. 2010: 23)

Die Schule ist beteiligt an der sozialen Hervorbringung und Konstruktion ethnischer Differenz, personifiziert in der Gestalt des ,Ausländers'/des ,Fremden'/des ,Migranten', die sich zu einer ethnischen Ordnung verdichtet. Organisationen, nicht nur die Schulen, produzieren durch ihre Distributionsund Selektionsleistungen die bestehende Sozialordnung und die zugehörige Unterordnung/Diskriminierung permanent selbst, um sie anschließend als 
objektive, quasi naturgegebene ethnische Ordnung zu erfahren, zu deuten und darzustellen. (Gomolla, Radtke 2002: 277)

Anstatt $\mathrm{zu}$ einer gerechteren Verteilung gesellschaftlicher Chancen zu führen, leistet Schule einen gewichtigen Beitrag zur Festschreibung der Unterscheidung zwischen Menschen mit und ohne Migrationsgeschichte. Die Ursachen für die Benachteiligung sind allerdings vielschichtig und durch das komplexe Zusammenwirken dieser Gründe kann es auch keine „einfachen“ Problemlösungen geben. (Dirim, Mecheril 2010: 123-136)

Die Praxis des Othering kann auf unterschiedlichen Ebenen im schulischen Kontext beobachtet werden, z. B.

- auf institutioneller Ebene: Durch die Lenkung der Schülerströme (Festsetzung der Einschulungskriterien, Einrichtung von Förderklassen, Abwicklung von Sonderschul-Aufnahme-Verfahren, Selektionsentscheidungen beim Übergang in die Sekundarstufe usw.) wird Selektion durchgeführt. Gomolla und Radtke (2002), die die institutionelle Diskriminierung in Deutschland untersucht haben, stellen fest, dass häufig ethnisierende Begründungen für organisatorisch-administrative Entscheidungen herangezogen werden, die letztlich negative Auswirkungen auf die Bildungskarrieren von Kindern mit Migrationsgeschichte haben.

- auf curricularer Ebene: In Österreich ist seit den frühen 1990er Jahren das (unverbindliche) Unterrichtsprinzip „Interkulturelles Lernen“ im Lehrplan verankert, das aufgrund der kulturellen Zuschreibungspraxis als veraltet gilt und derzeit überarbeitet wird. ${ }^{1}$ Interkulturelles Lernen fordert Sensibilität für kulturelle Differenzen und will verbindend zwischen den Kulturen wirken. Trotz der positiven Absicht erfolgt damit ungewollt eine Festigung ethnisierender Zuschreibungen. Schüler/innen mit Migrationsgeschichte werden oft automatisch - ohne Berücksichtigung ihrer individuellen Lebenssituation - auf eine „Herkunftskultur" festgelegt, selbst wenn sie in Österreich geboren und aufgewachsen sind. Hamburger (1999 zit. nach Dirim, Mecheril 2010: 142, 143) tritt für ein „situativ interkulturelles“ Lernen ein, da durch das dauerhafte Betonen und Hervorheben der Differenzen die bereits erreichten Grade an Gemeinsamkeiten und Übereinstimmungen übersehen werden. Interkulturelles Lernen werde sonst zur Dauerbelastung für migrationsandere Schüler/innen.

- auf Ebene von Lehrwerken: Welche Rollen, welche Zuschreibungen erfahren Migrationsandere in Schulbüchern? „Schulbücher spiegeln nicht die reale heterogene Gesellschaft wieder [sic], in ihnen sind Menschen mit Migrationsgeschichte nur in einem verschwindend geringen Umfang positiv handelnde Protagonist/innen." (Marschke; Brinkmann 2015: 387)

\footnotetext{
${ }^{1}$ Siehe Kommentar zum Unterrichtsprinzip Interkulturelles Lernen auf der Homepage des Bundesministeriums für Bildung und Frauen. https://www.bmbf.gv . at/schulen/unterricht/ uek/interkulturalitaet.html [25.2.2015]
} 
Eine Untersuchung von Binder (2013: 130, 131) von österreichischen Deutschbüchern der Unterstufe zeigt, dass Migrationsandere in den untersuchten Lehrwerken nur sehr spärlich vorkommen und häufig in Opferrollen sowie in niedrig klassifizierten Berufen dargestellt werden. Oder aber migrationsandere Menschen werden als Paradebeispiele für gelungene Integration präsentiert, wenn sie persönliche Defizite oder Misserfolge durch besondere Verdienste kompensieren und sich dadurch für die Mehrheitsgesellschaft nützlich erweisen. Unterschwellig werden so hierarchisierende Identifikationsangebote gemacht, die dazu beitragen sollen, dass die von der Mehrheitsgesellschaft gewünschten Werte, Ideologien oder Privilegien bestehen bleiben. (Binder 2013: 133) Nicht nur Schüler/innen, sondern auch Lehrer/innen werden durch Schulbücher mit Deutungsmustern aus dem Diskurs über Ausländer/ Migration/Fremde/Kulturkonflikt versorgt. (Gomolla, Radtke 2002: 286)

- auf Ebene der Lehrenden: Gut gemeinte Interventionen oder Fördermaßnahmen können auch dazu beitragen, „die Anderen“ im Klassenzimmer sichtbar zu machen und so unbeabsichtigt negative Wirkungen zu erzielen. Die Einstellung von Lehrkräften spielt nicht nur bei der Unterrichtsgestaltung, sondern auch in den Bereichen Elternarbeit, Laufbahnberatung, individuelle Förderung, Leistungsbeurteilung und im reflektierten Umgang mit Schulbüchern eine entscheidende Rolle.

\section{Zur dramapädagogischen Arbeit mit dem Text „In Between. Mein Leben in zwei Kulturen" von Amina Mahdy}

\subsection{Zur Wahl der Methode}

Migrantinnen und Migranten, Flüchtlinge und Menschen, die anders aussehen, nicht vertraut erscheinen oder von denen man erwartet, dass sie fremd sind, teilen größtenteils eine Erfahrung: Sie werden „anders“ behandelt. Dabei spielt es primär keine Rolle, ob eine positivere oder eine negativere Behandlung die Folge ist. Häufig ist jedoch die negative Variante, die mit einer Abwertung verbunden ist. Diese andere Behandlung ist häufig nicht messbar, aber durchaus fühlbar für die Personen, die sie erfahren. Andere, die der Mehrheitsgesellschaft zugehörig sind und dem äußeren Erscheinungsbild nach einer immer neu festzulegenden Norm entsprechen, kennen diese Erfahrungen nicht. [...] Oftmals sind die Erfahrungen der Diskriminierten jedoch nicht vorstellbar und nachvollziehbar. (Marschke; Brinkmann 2015: 381)

Um ein kleines Stück weit zu erfahren, wie es sich anfühlt, als „Andere/ $\mathrm{r}^{\text {“ }}$ behandelt zu werden, verwende ich den autobiografischen Text „In Between. Mein Leben in zwei Kulturen" von Amina Mahdy in meinem Seminar. In der Erzählung werden mehrere Erlebnisse geschildert, in denen die IchErzählerin als Kind und Jugendliche als Migrationsandere behandelt wird. Mit 
dramapädagogischen Methoden werden - nach themengeleiteten Warm-Ups - einige dieser Handlungssituationen aufgegriffen, um sie spielerisch aus der Perspektive einer/eines Betroffenen erlebbar zu machen.

Die zur Didaktisierung des Texts eingesetzten dramapädagogischen Methoden und Übungsformen haben ihre Wurzeln im britischen Drama in Education. Die entsprechenden Unterrichtskonzepte und Methoden sind z. B. nachzulesen in Schewe (2010, 2000, 1993), Neelands und Goode (2000) und Scheller (2006). Kennengelernt habe ich viele dieser Methoden im Rahmen des Hochschullehrgangs „Methode Drama - Drama- und Theaterpädagogik für Schule und Unterricht" an der Kirchlich-Pädagogischen Hochschule Wien/Krems (2010-2014) sowie in Seminaren von Manfred Schewe und Jonothan Neelands.

\subsection{Autorin und Text}

Die dramapädagogische Arbeit mit dem Text „In Between. Mein Leben in zwei Kulturen" (siehe Anhang) der jungen österreichischen Autorin Amina Mahdy (2013: 125-137) ${ }^{2}$ nehme ich in meinem Seminar für angehende Lehrkräfte als Ausgangspunkt zur Reflexion über (Alltags-)Rassismus und Othering. Amina Mahdy beschreibt in ihrem autobiografischen Text Alltagserfahrungen ihrer Kindheit und Jugend, in denen sie immer wieder zur „Anderen“ gemacht worden ist. Die Autorin wurde 1995 in Wien geboren und ist zweisprachig in Österreich aufgewachsen: Arabisch ist ihre Vatersprache, Deutsch ihre Muttersprache und die Sprache, in der sie hauptsächlich schreibt.

In Mahdys Text werden rassistische Handlungen vor allem durch die Gewalt der Sprache ausgeübt. Auch wenn Worte nicht körperlich verletzen können, können sie „treffen“ und „Wunden zufügen“, die durch die Macht des dahinter stehenden gesellschaftlichen Diskurses verstärkt werden.

... sprachliche Verletzungen [sind] nicht der subjektiven Empfindlichkeit der Adressaten geschuldet [...], sondern Effekte einer sozialen Praxis und von gesellschaftlichen Asymmetrien. Der Szene der Äußerung liegt eine ssoziale Grammatik zu Grunde, durch welche die verletzende Kraft sprachlicher Gewalt zu Stande kommt. (Herrmann; Kuch 2007: 13)

Die Autorin, die aufgrund ihrer Erfahrungen ein breites Spektrum rassistischer Diskriminierung zur Verfügung stellt, spricht mit ihrem Text nicht nur für sich, sondern für viele Menschen. Sie befreit sich dadurch aus der Position jener, die „besprochen werden“. Indem sie die Leser/innen an ihren Erfahrungen teilhaben lässt, macht sie Diskriminierungen sichtbar und sensibilisiert für eine diskriminierende Sprache, hinter der ein gesellschaftlicher Diskurs steht und die daher so wirkungsvoll ist.

\footnotetext{
${ }^{2}$ Amina Mahdy hat mit diesem Text, der 2013 in der Anthologie „preistexte 13“ veröffentlicht wurde, einen Literaturpreis der edition exil gewonnen. exil ist ein Kulturzentrum mit Schwerpunkt Literatur- und Kulturpräsentationen der Minderheiten und speziell der Roma in Österreich, welches Arbeiten von Künstler/innen und Künstlern fördert, die nach Österreich zugewandert sind oder einer ethnischen Minderheit angehören. http://www.zentrumexil.at/
} 
Die Erzählung beginnt mit einem Sandkastenerlebnis, bei dem die IchErzählerin zum ersten Mal die Erfahrung macht, „anders“ zu sein, indem zwei Mädchen mit rassistischen Bemerkungen ihr Spiel stören und ihr und ihrem Vater die Legitimität der Anwesenheit am Ort des Geschehens absprechen.

Doch auch Äußerungen und Handlungen, die auf den ersten Blick nicht rassistisch scheinen und vielleicht nicht so gemeint sind, können rassistisch wirken. Manche Leute sind „übermäßig nett“ zu der Protagonistin und in diesem Zusammenhang sei die harmlos anmutende Frage „Woher kommst du?“ angeführt, die durchaus ambivalenten Charakter hat: Einerseits suggeriert sie Interesse, andererseits macht sie jedoch deutlich, dass die angesprochene Person nicht in das erwartete Normalitätsskript passt, also als „fremd“ wahrgenommen wird, und ihre Anwesenheit erklärungswürdig ist. (Çiçek et al., in Marschke; Brinkmann 2015: 143) In eine ähnliche Kategorie fallen Komplimente und durchaus positive Zuschreibungen, die die Andersheit hervorheben wie zum Beispiel, dass gerade „Mischlingskinder besonders hübsch“ wären. Die selbstverständliche Zugehörigkeit zur Gesellschaft wird durch Aussagen dieser Art untergraben: Der Mangel der Nicht-Vollwertigkeit soll durch freundliche oder süßliche Rede ausgeglichen werden. Vor allem die ständige Wiederholung dieses zugewiesenen Status schreibt das Gefühl der Nicht-Zugehörigkeit sozial fest und verletzt tiefgehend.

Mehrfach taucht im Text die Versicherung auf, dass man „absolut nichts gegen Ausländer habe", vor allem dann wenn sie so ein gutes Benehmen hätten, dass sich sogar "die Unsrigen“ etwas davon abschauen könnten. Die verletzende Rede tarnt sich hier als vermeintliches Lob, das in der Unterscheidung zwischen einem „Wir“ und einem „Nicht-Wir“ mündet und seine Kraft aus dem Kollektiv bezieht.

Sprachliche Gewalt entfaltet sich nicht einfach durch ihre Semantik, sondern ebenso durch die Kraft, die mit ihr kommuniziert wird. Es ist beispielsweise entscheidend, ob ich jemanden als Individuum missachte oder im Namen einer gesellschaftlich legitimierten Instanz. Insofern sich das Sprechen also in machtvolle Diskurse einzuschreiben vermag, ist es nicht allein Träger von Bedeutung, sondern vielmehr in der Lage, die ganze hierarchische Kraft einer Gesellschaft und ihrer Geschichte aufzurufen und gegen seine Adressatin zu wenden. (Herrmann; Kuch 2007: 12)

Dazu kommt die offene Verwendung von abwertenden Bezeichnungen. In der Erzählung verwendet eine Lehrkraft im schulischen Musikunterricht zum Beispiel die Bezeichnung „Negermusik“ für Jazz und in einer Krankenhausszene fürchten Patientinnen das Kommen einer „Ausländerin“, als sie einen nichtdeutschsprachigen Namen am Nachbarbett lesen. Damit ist ein weiteres Kennzeichen rassistischer Rede markiert: Die Sprecher/innen implizieren bewusst oder unbewusst ein soziales Gefälle und maßen sich die Autorität an, über Andere herabwürdigend zu sprechen. Dabei greifen sie auf soziale diskursive Praxen zurück. (Çiçek et al., in: Marschke; Brinkmann 2015: 155, 156)

Als Rechtfertigung der Diskriminierung kommen Vorurteile und stereotype Denkweisen einzelner, aber auch Traditionen, Gewohnheiten und 
Gesetze in Betracht, die das soziale Handeln von Kollektiven strukturieren. Akteure können Einzelne, kleine Gruppen oder auch Organisationen sein, in denen bestimmte Unterscheidungen bzw. Unterscheidungspraxen bereits institutionalisiert sind. Sie müssen Gelegenheit haben und über Definitionsmacht verfügen. Die Objekte/Opfer müssen Merkmale haben, die sie diskriminierbar machen und eine sozial folgenreiche Ungleichbehandlung ermöglichen. Insofern setzt Diskriminierung eine asymmetrisch angelegte Interaktionssituation voraus, in der soziale Ordnungen hergestellt und fortgeschrieben werden. (Gomolla; Radtke 2002: 16)

Das Repertoire der Missachtung zeigt, dass die Bezeichnung äußerer Merkmale mitunter skurrile Formen annehmen kann. Die Ich-Erzählerin wehrt sich beispielsweise gegen folgende Zuschreibung: „Farbig' finde ich übrigens immer ein wenig seltsam. Farbig ist für mich bunt, und dass ich bunt bin, finde ich nicht."

Die Grenze zwischen verbaler und körperlicher Gewalt kann rasch verschwimmen, wie die Begegnung des zwölfjährigen Mädchens mit einem Betrunkenen auf der Straße deutlich macht: Das Mädchen wird gestoßen und beschimpft: „Schleich di! Geh hin, wo'st herkummst. Mir brauchen eich do net. Host g' heat?" Der Ich-Erzählerin wird ihr Subjektstatus abgesprochen wie auch die Legitimität ihrer Anwesenheit an diesem Ort. Sie wird mit dem Vorurteil, der unerwünschten Gruppe der „Ausländer“ zuzugehören, weggewiesen. Dabei ist es unerheblich, inwieweit die Adressatin dieser Gruppe tatsächlich angehört. Die Handlung kann als „gruppenbezogene Menschenfeindlichkeit“ eingestuft werden: Menschen werden aufgrund der zugewiesenen Gruppe, die als „fremd“, anders „unnormal“ markiert ist, als ungleichwertig betrachtet und in Folge mit Abwertung, Diskriminierung, Ausschluss oder Gewalt konfrontiert. (Zick; Küpper, in: Marschke; Brinkmann 2015: 95)

\subsection{Dramapädagogische Arbeit mit dem Text „In Between“}

Noch bevor die Erzählung in der Seminargruppe gelesen wird, gibt es thematische Warm-ups und Übungen, die zur Arbeit mit dem Text hinführen. Je nach Gruppengröße, Gruppenzusammensetzung und der Vertrautheit der Teilnehmer/innen mit dramapädagogischen Unterrichtsmethoden, wähle ich aus folgenden Aktivitäten aus:

Warm-up (zur Wahl) -

- Gruppenbildung: Gruppen konstituieren sich nach vorgegebenen äußeren Merkmalen (Augen, Beine, Haare, usw.) und definieren dabei selbst das konstituierendeElement, z.B.: hochgesteckte/gefärbte/lange/kurze/frisch gewaschene/ ... Haare oder müde/blaue/braune/grüne/bebrillte/ ... Augen. In einem zweiten Schritt werden die gruppenkonstituierenden Merkmale frei gewählt und können auch den Charakter vorerst „unsichtbarer" Zuordnungen haben. Das verbindende Element soll durch die Gruppenteilnehmer/innen dargestellt und von den anderen erraten 
werden. (z. B. Gruppe der Hundebesitzer, der Vegetarier/innen, der Liebhaber/innen romantischer Sonnenuntergänge usw.)

- Aufwachen: Die Teilnehmer/innen liegen in Embryostellung auf dem Boden und wachen zu langsamer Musik in einer fremden Umgebung auf. Langsam beginnen sie, mit dieser Umgebung und auch untereinander Kontakt aufzunehmen.

- Gruppen bilden: Ca. 6 - 7 Personen bilden einen engen Kreis und fassen sich an den Händen. Eine Person versucht, von außen in den Kreis einzudringen.

- Party: Kleingruppen stehen beisammen, ein/e Spieler/in geht von Gruppe zu Gruppe und findet nirgends Anschluss. Diese Rolle wird mehrfach vergeben. Anschließend erfolgt eine kurze Reflexion.

- Statusübung: Die Teilnehmer/innen werden in zwei Gruppen (A, B) geteilt. Zuerst befindet sich Gruppe A im Hochstatus und Gruppe B im Tiefstatus. Teilnehmer/innen der Gruppe B senken den Blick, wenn sie jemandem aus Gruppe A begegnen. Anschließend erfolgen ein Rollenwechsel sowie eine kurze Reflexion.

Vor dem Lesen des Textes -

- „Gerüchte“: Gruppen bereiten Gerüchte über Personen oder Personengruppen vor und kolportieren sie. Durch die Lehrperson in Rolle können Informationen über „die Anderen“ eingestreut werden.

- „Kollektive Rolle“: Welche abfälligen Bemerkungen über Fremde oder fremd aussehende Menschen hast du schon gehört? Diese Bemerkungen werden zu einem in der Mitte stehenden leeren Sessel gesprochen.

„Aufführungskarussell“ — Die Geschichte wird in fünf Einzelepisoden zerlegt, separat kopiert und auf fünf Gruppen aufgeteilt ${ }^{3}$ :

1. Sandkastenepisode

2. Schulepisode (Englischbuch)

3. Episode mit Mann aus Gasthaus („Schleich di!")

4. Krankenhausepisode

5. Tiergartenepisode (Picknick)

\footnotetext{
${ }^{3}$ Der Text „In Between. Mein Leben in zwei Kulturen“ von Amina Mahdy ist in Anhang A nachzulesen. Die fünf Episoden sind im Originaltext durch Anmerkungen in eckigen Klammern nummeriert und ausgewiesen.
} 
Die jeweilige Gruppe liest die ihr zugeteilte Episode und entwickelt ein Standbild dazu, das durch „Thought tracking“ belebt wird: Die Rollen befinden sich in eingefrorener Position und reagieren auf einen Berührungsimpuls, indem sie ihre momentanen Gedanken hörbar äußern. Das Bild kann eventuell auch in Zeitlupe weiterentwickelt werden. Gleich anschließend wird „Amina“ gefragt, wie es ihr nach dem jeweiligen Erlebnis gehe. Sie sitzt auf einem Sessel in der Mitte und die anderen in ihrer Gruppe stehen hinter ihr und sprechen ihre Gedanken als inneren Monolog (= „kollektive Rolle").

Die Krankenhausepisode kann humorvoll als Rollenspiel bearbeitet werden, die beiden "Quasseltanten“ können überzeichnet werden. Die Personen im Krankenzimmer führen ein Gespräch mit jeweils einem „Alter ego“ hinter sich. (Was sprechen die Personen aus und was denken sie sich wirklich? Der Unterschied zwischen den nach außen hin getätigten Aussagen und dem „Subtext" der Figur wird durch diese Technik aufgezeigt. - Komik ist an dieser Stelle durchaus erwünscht.)

Der Absatz, in dem zur Diskussion steht, ob die Familie vor Schulbeginn nach Ägypten übersiedelt, wird erst an dieser Stelle gelesen und dann spielend erfahren:

- „Ratgeberkreis“: „Amina“ hat von den Überlegungen (Wünschen) der Eltern gehört und berät sich im Kreise ihrer Freundinnen.

- „Gedanken- bzw. Argumentationsallee“ zum Thema Übersiedeln nach Ägypten: „Amina“ geht durch das von den Teilnehmer/innen gebildete Spalier und hört von der einen Seite die Argumente der Eltern und von der anderen Seite ihre eigenen Gedanken und Ängste. Am Ende der Allee trifft sie für sich eine Entscheidung.

- Eventuell: „Amina“ vertraut einer Lehrerin an, dass sie nicht mit ihrer Familie nach Ägypten ziehen will. Es folgt ein Gespräch der Eltern mit der Lehrkraft.

Abschluss -Zum Schluss wird der Ganztext gelesen.

- „Marking the Moment“: Jede/r Teilnehmer/in überlegt, an welcher Stelle er/sie sich am besten mit „Amina“ identifizieren konnte, sucht sich dafür einen Platz im Raum und nimmt die entsprechende Position ein. Eventuell sagen die Teilnehmer/innen einen Gedanken oder geben ein Geräusch von sich.

- „Danke-Kreis“: Die Teilnehmer/innen stehen im Kreis und verabschieden sich, indem sie Blickkontakt mit allen aufnehmen und still „danke“sagen.

\subsection{Reflexion der Unterrichtssequenz}

Die performative Arbeit mit literarischen Texten hat sich in meiner Unterrichtspraxis vielfach als nachhaltig herausgestellt. Besonders beim Thema Othering 
führen der erlebte Zugang und der damit verbundene Perspektivenwechsel zu einer tieferen Auseinandersetzung mit der Materie, die Betroffenheit erzeugt. Durch den ganzheitlichen Zugang wird Raum für die Thematik des Othering geschaffen. Das Thema wird nicht nur diskursiv behandelt, sondern aus der Perspektive „Drama“ heraus eine Erfahrung ermöglicht, die Empathiefähigkeit fördert und durch (Selbst-)Reflexivität die pädagogische Professionalität vertieft. Ohne die dramapädagogische Arbeit mit dem Text von Amina Mahdy haben einige Studierende in vorangegangenen Semestern m.E. manchmal lediglich den Ausdruck sprachlicher Korrektheit hinter der Thematisierung der Praxis des Othering gesehen. Dass sprachliche Praxen jedoch eng mit dem Aushandeln hegemonialer Verhältnisse verbunden sind, kann durch den dramapädagogischen Zugang ein Stück weit erfahren werden.

Da es sich bei der Lehrveranstaltung um ein Wahlfach handelt, wird es von Studierenden besucht, die in der Regel großes Interesse am Bereich Deutsch als Zweitsprache haben. Auf offene rassistische Äußerungen bin ich in diesem Rahmen noch nie gestoßen. Allerdings kann durchaus Diskussionsbedarf bezüglich alltagsrassistischer Sprache und (institutioneller) Diskriminierung aufkommen. Dazu zählen zum Beispiel kulturalistische Zuschreibungen, in denen die schulische Leitungsbereitschaft und das Ausmaß der Unterstützung durch das Elternhaus mit „kulturellen“Faktoren in Verbindung gebracht werden. Der Blick durch die „Kulturbrille“ verstellt dabei häufig die Sicht auf sozioökonomische Faktoren, migrationsbedingte Prägungen und individuelle Voraussetzungen der Lernenden. (Vgl. Foitzig 2013) Zudem werden schulische Benachteiligungen im Zusammenhang mit „Deutsch als Zweitsprache“ thematisiert und die Rolle von Schule in der Migrationsgesellschaft wird diskutiert.

Grundsätzlich habe ich mit dieser Unterrichtssequenz sehr gute Erfahrungen gemacht: Einerseits sorgt die theoretische Aufarbeitung des Themenbereichs für Information und den entsprechenden Fokus, andererseits haben die Studierenden im dramapädagogischen Teil die Möglichkeit, sich selbst einzubringen und es gibt Raum für Reflexion. Die Bereitschaft, sich aktiv handelnd mit dem Themengebiet auseinanderzusetzen, war groß und bot für die Studierenden eine willkommene methodische Abwechslung im Studienalltag.

Bei der Durchführung der Unterrichtseinheit ist es aus Gründen der Gruppendynamik wichtig darauf zu achten, dass die Gruppe nicht zu klein und nicht zu groß ist. (Ideal sind 15 bis 20 Personen.)

\section{Abschließende Bemerkungen}

In diesem Beitrag habe ich in einem ersten Schritt versucht, die gesellschaftliche Praxis des Othering theoretisch zu umreißen sowie die Bedeutung und die Implikationen für den schulischen Alltag herauszustellen. Es ist nicht einfach, Othering zu vermeiden, da viele Prozesse unbewusst ablaufen und der Struktur sowie der Funktionsweise der Institution Schule geschuldet sind. Ein Blick auf die schulische Realität an einem Standort kann zum Beispiel Aufschluss darüber geben, ob die gebotenen Rahmenbedingungen dazu beitragen, Ungleichheiten 
festzuschreiben. Selbst versierten und engagierten Pädagog/innen kann es passieren, unbeabsichtigt ausgrenzend zu wirken, indem beispielsweise durch interkulturelles Arbeiten oder der Einteilung von Lerngruppen Zuschreibungen gefördert werden. Es bedarf großer Aufmerksamkeit und Sensibilität, um auf der Vielzahl schulischer Handlungsebenen Othering zu vermeiden.

In einem zweiten Schritt erfolgte die dramapädagogische Aufbereitung des Texts von Amina Mahdy, welche verdeutlichen sollte, wie Othering aus der Perspektive einer Betroffenen erfahren werden kann.

Ziel der Arbeit im Seminar war die Sensibilisierung für sprachliche Gewalt, um einen Beitrag zur Prävention von Diskriminierung und Rassismus zu leisten, zum Hinschauen zu motivieren sowie zur individuellen und institutionellen Selbstreflexion („ethnisches Monitoring“) anzuregen.

Patentrezepte für professionelles pädagogisches Handeln in heterogenen Gruppen von Lernenden kann es freilich keine geben, vielmehr gilt es, sich eine offene, reflexive Haltung zu eigen zu machen, Unterschiede wahrzunehmen und gleichzeitig Menschen nicht darauf festzulegen.

Schließlich möchte ich mich noch herzlich bei Amina Mahdy und dem exil Verlag (Geschäftsführung: Christa Stippinger) für die freundliche Genehmigung bedanken, dass sie den Text „In Between. Mein Leben in zwei Kulturen“ allen Leser/innen dieses Beitrags zur Verfügung stellen (siehe Anhang).

\section{Bibliografie}

Ahrenholz, Bernd \& Oomen-Welke I. (2010) (Hrsg.): Deutsch als Zweitsprache. (2. Aufl.). Baltmannsweiler (= Handbuch zur Didaktik der deutschen Sprache und Literatur, Bd. 9)

Auernheimer, Georg (Hrsg.) (2010): Interkulturelle Kompetenz und pädagogische Professionalität (3. Aufl.). Wiesbaden: Verlag für Sozialwissenschaften (= Interkulturelle Studien Bd. 13)

Binder, Nicole (2013): Durch migrationsgesellschaftliches Othering erzeugter Pseudo-Paternalismus in Deutschsprachlehrwerken. Universität Wien: Diplomarbeit http://othes.univie.ac.at/27650/1/2013-04-09_0800383.pdf [zuletzt aufgerufen am 13. Januar 2015]

BMBF (2014): Schüler_innen mit anderen Erstsprachen als Deutsch. Statistische Übersicht. Schuljahre 2006/07 bis 2012/13.

Informationsblätter des Referats für Migration und Schule 2 / 2013-14, Wien. http://www.schule-mehrsprachig.at/fileadmin/schule_ mehrsprachig/redaktion/Hintergrundinfo/info2-13-14.pdf [zuletzt aufgerufen am 7. August 2015]

Çiçek, Arzu; Heinemann, Alisha \& Mecheril, Paul (2015): Warum so empfindlich? Die Autorität rassistischer Ordnung oder ein rassismuskritisches Plädoyer für mehr Empfindlichkeit. In: Marschke, Britta 
\& Brinkmann, Heinz Ulrich (Hrsg.): Ïch habe nichts gegen Ausländer, aber...". Alltagsrassismus in Deutschland. Münster: LIT

Dirim, Inci \& Mecheril, Paul (2010): Die Schlechterstellung Migrationsanderer. Schule in der Migrationsgesellschaft. In: Mecheril, Paul et al.:

Migrationspädagogik. Weinheim, Basel: Beltz, 121-149

Dirim, Inci; Mecheril, Paul \& Melter, Claus (2010): Umgang mit migrationsbedingter Heterogenität im österreichischen Bildungssystem. In: Mecheril, Paul et al.: Migrationspädagogik. Ergänzende Materialien. Weinheim, Basel: Beltz, 22-24

Foitzig, Andreas (2013): Interkulturelle Kompetenz. In: ajs Kompaktwissen. http://www.ajs-bw.de/media/files/aktuell/2013/KWInterkultur2013_L10.pdf [zuletzt aufgerufen am 7. August 2015]

Gogolin, Ingrid (2008): Der monolinguale Habitus der multilingualen Schule. Münster: Waxmann

Gomolla, Mechtild \& Radtke, Frank-Olaf (2002): Institutionelle Diskriminierung. Opladen: Leske und Budrich

Herrmann, Steffen Kitty; Krämer, Sybille \& Kuch, Hannes (Hrsg.) (2007): Verletzende Worte. Die Grammatik sprachlicher Missachtung. Bielefeld: transcript verlag

Mahdy, Amina (2013): „In Between“- Mein Leben in zwei Kulturen. In: Stippinger, Christa (Hrsg.): anthologie: preistexte 13. Wien: edition exil, 125-141

Marschke, Britta \& Brinkmann, Heinz Ulrich (Hrsg.) (2015): Ïch habe nichts gegen Ausländer, aber...". Alltagsrassismus in Deutschland. Münster: LIT

Mecheril, Paul; Castro Varela, Maria do Mar; Dirim Inci; Kalpaka, Annita; Melter, Claus (2010): Migrationspädagogik. Weinheim, Basel: Beltz

Mecheril, Paul (2002): „Kompetenzlosigkeitskompetenz“. Pädagogisches Handeln unter Einwanderungsbedingungen. In: Auernheimer, Georg (Hrsg.): Interkulturelle Kompetenz und pädagogische Professionalität. Opladen: Leske u. Budrich, 15-34

Mikura, Raphaela (2013) Mehrsprachigkeit ohne Othering? Diplomarbeit, Universität Wien http://othes .univie.ac.at/25062/ [zuletzt aufgerufen am 7. August 2015]

Müller, Thomas (2008): Dramapädagogik und Deutsch als Fremdsprache. Eine Bestandsaufnahme. Saarbrücken: Müller

Neelands, Jonothan \& Goode, Tony (2000) (eds.): Structuring Drama Work. A Handbook of available forms in theatre und drama. Cambridge: Cambridge University Press

ÖDAF Mitteilungen (2010): Informationen des Vereins „Österreichischer Verband für Deutsch als Fremdsprache/Zweitsprache“. Vielfalt - Sprachen Identität(en). Kontinuität und Veränderung im Kontext DaF/DaZ (Heft 1)

Scheller, Ingo (2006): Szenisches Spiel. Handbuch für die pädagogische Praxis. Berlin: Cornelsen Scriptor 
Schewe, Manfred (2010): Drama- und Theaterpädagogik im Deutsch als Fremd- und Zweitsprache-Unterricht. In: Krumm, Hans Jürgen et al. (Hrsg.): Deutsch als Fremd- und Zweitsprache. Ein internationales Handbuch (2. Halbband). Berlin, New York: Walter de Gruyter, 1589-1595

Schewe, Manfred (2000): DaF-Stunden dramapädagogisch gestalten - wie mache ich das? In: Schlemminger, Gerald; Brysch, Thomas \& Schewe Manfred. (2000) (Hrsg.): Pädagogische Konzepte für einen ganzheitlichen DaF-Unterricht. Berlin: Cornelsen, 72-105

Schewe, Manfred (1993): Fremdsprache inszenieren. Zur Fundierung einer dramapädagogischen Lehr- und Lernpraxis. Oldenburg: Didaktisches Zentrum

Schmitt, Tom \& Esser, Michael (2010): Status-Spiele. Wie ich in jeder Situation die Oberhand behalte. Frankfurt: Fischer

Schweiger, Hannes (2006): Schreiben in Zwischenräumen. Literatur der Migration. In: Kurswechsel 2, 44-53.

http: //www . beigewum.at/wordpress/wp-

content/uploads/044_hannes_schweiger.pdf [zuletzt aufgerufen am 7 . August 2015]

Zick, Andreas \& Küpper, Beate (2015): Alltagsdiskriminierung als Form der Gruppenbezogenen Menschenfeindlichkeit. In: Marschke, Britta \& Brinkmann, Heinz Ulrich (Hrsg.): Ïch habe nichts gegen Ausländer, aber...

. Alltagsrassismus in Deutschland. Münster: LIT, 93-115 
Mahdy, Amina (2013): „In Between“ - Mein Leben in zwei Kulturen. In: Stippinger, Christa (Hrsg.): anthologie: preistexte 13. Wien: edition exil, 125-137

Das erste Mal, als ich draufkam, dass mit mir etwas nicht stimmt, da war ich - keine Ahnung - auf jeden Fall noch nicht in der Schule. Also fünf oder so. Bis dahin bin ich mir ganz normal vorgekommen. Inzwischen bin ich siebzehn und eigentlich komme ich selbst mir immer noch ganz normal vor. Es ist nur so, dass es bei mir etwas gibt, das einigen anderen nicht normal vorkommt. Eindeutig nicht, das hab ich immer wieder erfahren. Da kann ich machen, was ich will. Verstehen tu ich es aber noch immer nicht so richtig.

Ich erzähle einfach mal, wie das so ist bei mir. Vielleicht komme ich dann ja drauf oder jemand anders kann es mir erklären. Am besten fange ich mit meinem allerersten Schlüsselerlebnis an. Da hat sich Folgendes ereignet:

[1: Beginn Sandkastenepisode]

Es war ein ziemlich heißer Tag. Die Luft flimmerte und es duftete nach Lindenblüten. Papa und ich waren auf dem Spielplatz in der Nähe vom Naschmarkt. Er saß auf einer Bank im Schatten neben unseren Einkäufen, und ich baute im Sandkasten an einem Kanalsystem. Also das heißt, ich grub mehrere Rinnen, die ich miteinander verband und in die ich dann Wasser leiten wollte. Einen Kübel Wasser hatte ich mir schon bereitgestellt. Da stampften dicht neben meinen Händen plötzlich zwei Füße in mein Bauwerk und ließen den ganzen Sand, den ich ausgehoben hatte, wieder einsinken.

Ich war schon so weit gewesen, jetzt konnte ich von Neuem beginnen. Na toll! Ich erwartete, dass die Füße gleich verschwinden würden, doch das taten sie nicht. Sie rührten sich nicht von der Stelle, wackelten nur ein wenig mit den Zehen. Ich tupfte sie an und sagte irgendwas in der Art von: „He, geh weg! Du machst mir alles kaputt", aber die Füße blieben und wackelten als Antwort nur etwas schneller mit den Zehen. Da sah ich hoch und in das Gesicht eines Mädchens, das, sicher einige Jahre älter als ich, herausfordernd auf mich herabblickte. Sofort begriff ich: Das ist der nicht versehentlich passiert, die ist absichtlich reingetrampelt. Trotzdem machte ich noch einen Versuch: „Geh weg da. Du kannst woanders stehen. Siehst du nicht, dass ich da etwas baue?" Das Mädchen rührte sich nicht. Die Hände hatte es vor der Brust verschränkt und es blickte geradezu triumphierend zu mir herab. Neben ihm stand, wie ich erst jetzt bemerkte, ein zweites Mädchen in der gleichen Siegerpose. Die beiden gehörten offensichtlich zusammen und ebenso offensichtlich wollten sie mich ärgern.

„Warum machst du das?", fragte ich. „Ich hab dir doch nichts getan.“

Und dann fällt er. Der Schlüsselsatz vom Schlüsselerlebnis:

„Dein Papa soll sich waschen“, platzte die Erste heraus, die mit den Füßen noch immer knapp vor mir stand. Von der Grube war nichts mehr zu sehen.

„Dein Papa soll sich waschen“, echote die andere.

„Dein Papa ist schmutzig“, ergriff die Erste wieder das Wort. Einen ganz 
kurzen Augenblick lang wollte ich protestieren. Dass mein Papa ganz sicher nicht schmutzig sei, dass er SEHR sauber sei. Dass er sich immer wasche, dass sie selber ganz schwarze Zehennägel hätten und so weiter. Aber ich sagte nichts. Denn bevor ich den Mund aufmachen konnte, hatte ich verstanden, was sie meinten.

Sie meinten die Hautfarbe meines Vaters.

Ich kippte den Kübel mit dem Wasser über die Füße, die mein Bauwerk zerstört hatten, sammelte meine Schaufeln und Formen ein und verließ die Sandgrube. Eine der beiden rief mir noch nach:

„Du könntest dich auch mal waschen!“ Ich ging ganz langsam weg, es sollte nicht so aussehen, als würde ich vor ihnen davonlaufen, und drehte mich nicht mehr um.

Mein Vater las Zeitung und hatte von der Szene nichts mitbekommen. Für mich war er der liebste Papa der Welt. Ich erzählte ihm nichts von den Mädchen, sondern bemühte mich, dass er mir nichts anmerkte. Ich sagte nur:

„Yala, ya Baba!“ Das heißt: „Gehen wir, Papa!“ Ich rede nämlich Arabisch mit ihm. Er kommt aus Ägypten und er hat eine dunkle Hautfarbe.

Das haben die Mädchen gemeint mit schmutzig.

[1: Ende Sandkastenepisode]

Ich weiß nicht, ob mir bis zu dem Tag jemals bewusst war, dass mein Papa eine dunkle Hautfarbe hat, und dass ich selber auch dunkler bin als die Leute hier. Keine Ahnung. Jedenfalls hab ich an diesem Tag verstanden, dass es Leute gibt, die das als Fehler ansehen.

Im Kindergarten bin ich nicht aufgefallen wegen meinem Teint und auch nicht wegen dem von Papa. Da war es so, dass ich eine von ganz wenigen war, die Deutsch sprachen. Ich glaube, wir waren insgesamt nur vier mit deutscher Muttersprache. Ach ja, meine Mutter ist Österreicherin. Das heißt, in Österreich geboren und ihre Eltern auch und die Großeltern und so weiter. Darum spreche ich Deutsch und Arabisch und darum bin ich nicht ganz hell- und nicht ganz dunkelhäutig.

Papa ist inzwischen auch Österreicher. Das zählt aber nicht bei den Leuten. Weil er ja dunkel ist. Weil er nicht hier geboren ist, seine Eltern und Großeltern schon zweimal nicht.

Wenn wir zu dritt unterwegs sind, fallen wir auf. Irgendwann habe ich das bemerkt. Leute schauen uns an, mustern uns. Manche schauen uns böse an. In so einem Fall tu ich so, als würde ich es nicht merken.

Andere sind besonders nett zu uns. Übermäßig nett.

Am liebsten sind mir die, die uns ganz normal behandeln. So normal, wie sie Leute behandeln, die so aussehen wie sie selbst. Das kommt zum Glück auch vor.

Gemein sind ganz wenige. Ich meine so, wie die Mädchen im Sandkasten. Die fand ich schon gemein. Aber so was ist mir eher selten passiert.

[2: Beginn Schulepisode]

In den ersten drei Klassen Gymnasium hatte ich einen Mitschüler, der immer versuchte, mich zu ärgern. Er hat mir oft Sachen versteckt oder kaputt gemacht, 
meine Hefte und Bücher vollgeschmiert und dauernd blöde Bemerkungen gemacht über meine Haare oder meine Hautfarbe. Das hat mich zwar schon immer wieder geärgert, aber nicht übermäßig aufgeregt, weil auch andere Mitschüler mit dem Burschen Probleme hatten.

Aber eines Tages war mein Englischbuch verschwunden, und zwar wirklich unauffindbar. Ich bat alle nachzuschauen, ob sie es versehentlich eingesteckt hätten. Jeder durchwühlte sein Bankfach und seine Schultasche und versprach mir auch, bei sich zu Hause danach zu suchen, aber dieser Bursche grinste mich nur blöd an: „Als ob ich von dir etwas haben wollte.“

Ich hätte ihn daran erinnern können, wie oft ich schon alles mögliche Zeug von mir auf seinem Platz gefunden hatte, aber das wäre sinnlos gewesen. Außer einem blöden Gequatsche hätte mir das nichts eingebracht.

Schließlich fand ich mich damit ab, dass das Buch verloren war. Verdächtigt habe ich niemanden. Wer sollte schon ein Buch von einem Mitschüler nehmen, wo doch jeder selbst eines hat? Wohl oder übel musste ich mir ein neues kaufen - von meinem Taschengeld, wohlgemerkt, weil Mama meine "Schlamperei“ nicht unterstützen wollte, wie sie mir wortreich erklärte.

Und dann, ein paar Tage danach, sah ich mein altes Englischbuch auf dem Tisch meines speziellen Freundes liegen.

„Du hast mein Englischbuch!“, fuhr ich ihn an. „Ich hab mir eine neues kaufen müssen, das kannst du mir jetzt zahlen!“

Da machte er eine freche Grimasse und sagte voll Verachtung: „Wasch du dir erst einmal die Scheiße aus dem Gesicht." Und für den Fall, dass es nicht bei mir angekommen war, wiederholte er den Satz. Es war aber schon beim ersten Mal angekommen, und wie es gemeint war, habe ich diesmal gleich kapiert. Ich war auch nicht mehr so überrascht wie damals bei den Mädchen im Sandkasten, dafür aber noch verletzter.

Erst ein paar Tage später konnte ich mit Mama darüber reden.

[2: Ende Schulepisode]

Was ist so schlimm daran, dass meine Haut nicht rosig oder käseweiß ist? Ich verstand es nicht und ich verstehe es bis heute nicht.

Es gibt allerdings auch viele, die sagen, sie würden mich um meinen Teint beneiden.

Jetzt haben wir übrigens eine tolle Klassengemeinschaft. Dieser Junge ist jetzt nicht mehr in unserer Klasse. Er ist sitzen geblieben.

Es ist jetzt nicht wirklich Thema bei uns, wer woher kommt oder wer wie ausschaut. Wir machen schon Witze darüber, aber niemand ist verletzend. Das dunkelhäutigste Mädchen in der Klasse und ich haben irgendwann den Spruch eingeführt: „, . , nur weil wir schwarz sind. “Den wenden wir an, sobald jemand etwas von uns will oder sich einen Scherz mit uns erlaubt. Natürlich gibt es davon auch die Umkehrung: „..., weil ihr schwarz seid!“

In beiden Fällen ist das aber ausschließlich eine Blödelei, die nur jemanden aufhorchen lässt, der unsere Klasse nicht kennt.

Spaß haben wir auch, wenn mal wieder ein neuer Lehrer versucht unsere Namen, die aus vielen Ländern stammen, richtig auszusprechen, was oft 
gründlich danebengeht.

Die Lehrer machen keinen Unterschied, wie sie uns behandeln, außer dass sie mit der Hautfarbe mitunter Probleme der anderen Art haben. Davon werde ich später erzählen. Bei einer Lehrerin, die im Zusammenhang mit Jazz zum Beispiel sehr abfällig über „Negermusik“ sprach, war ich nicht gerade „Liebling“, aber einigermaßen korrekt hat sie mich schon behandelt. Inzwischen ist sie pensioniert.

Also das Wort „Negermusik“ stört mich, weil es immer abwertend gebraucht wird und ich außerdem sehr viel von der Musik halte, die damit gemeint wird. Das Wort „Neger“ hingegen finden übrigens weder Papa noch ich so schlimm wie viele Leute hier. Wichtig ist mir, wie mich jemand behandelt, nicht wie er mit Wörtern jongliert, wie Dunkelhäutige, Farbige, Menschen mit Migrationshintergrund und was es da alles gibt.

„Farbig“ finde ich übrigens immer ein wenig seltsam. Farbig ist für mich bunt. Und dass ich bunt bin, finde ich nicht.

Man kann jedes Wort verächtlich sagen. Einen Eiertanz um Wörter finde ich eher peinlich. Ich habe mich schon gefragt, wie ein Behinderter wohl die Bezeichnung „Mensch mit besonderen Bedürfnissen“ schätzt. Das kommt mir so ähnlich vor.

Ich habe kein Problem, einen „Mohr im Hemd“ zu bestellen, so wie die Wiener „Frankfurter“ oder die Vorarlberger „Wienerle“ jausen.

Aber was ich einmal in einem großen Bregenzer Supermarkt gesehen habe, fiel für mich in eine andere Kategorie. Da hing in der Feinkostabteilung ein Plakat mit der Aufschrift: „So mögen wir die Wiener!“ Darunter war ein großes Wiener Schnitzel abgebildet.

[6: Übersiedlung nach Ägypten]

Bevor ich in die Schule kam, also ich meine, bevor ich überhaupt eingeschult wurde, haben mich meine Eltern einmal sehr ernsthaft gefragt, was ich davon hielte, wenn wir nach Ägypten übersiedelten.

Meine Antwort stand sofort fest: Nichts!

Das Thema kam dann einige Zeitlang immer wieder zur Sprache, und ich hatte das unangenehme Gefühl, dass mich beide umzustimmen versuchten.

Mama, die keinen Winter mag, warb unter anderem damit, dass wir dann das ganze Jahr über schönes Wetter hätten und wir die Sommerferien, falls es uns zu heiß würde, ja in Österreich verbringen könnten.

Papa rief mir lustige Ferientage in Erinnerung und wie sehr ich bei all meinen vielen Verwandten immer willkommen war und überall Spielgefährten vorgefunden hatte.

Ich habe nämlich keine Geschwister, und als ich klein war, habe ich das sehr bedauert.

In Ägypten, egal bei welcher Familie wir auf Besuch waren, gab es überall Kinder in allen Altersstufen. Es war immer lustig und ich habe mir sehr gewünscht, dass ich das in Wien auch hätte.

Aber deswegen nach Ägypten übersiedeln? Das war für mich ganz unvorstellbar. Schließlich hatte ich hier auch meine Freunde und ich liebte den 
Winter.

Doch meine Eltern ließen nicht so schnell locker.

Schließlich, als ich begriff, dass sie die Idee ganz konkret weiterverfolgten, dass sie zum Beispiel Unterlagen über deutsche Schulen in Kairo studierten, wurden mir diese Gespräche unangenehm. Eines Tages erklärte ich dann ein wenig theatralisch:

„Ich bin in Wien geboren. Ich bin eine Wienerin. Ich mag Ägypten und mag auch Bregenz (da ist Mama her), aber ich liebe Wien. Wien ist meine Heimatstadt."

Ich glaube, nach dieser deutlichen Deklaration war das Thema dann ein für alle Mal vom Tisch. Jedenfalls hörten meine Eltern auf, mich damit zu bedrängen.

Und wenn mich, was besonders in Ägypten immer wieder der Fall ist, andere Leute fragen, ob ich denn nicht vielleicht lieber in Kairo leben würde, dann zitieren entweder Papa oder Mama meine Erklärung, weshalb sich diese auch bis heute im Wortlaut erhalten hat. Auf Arabisch und auf Deutsch.

Seitdem habe ich oft in Ägypten Urlaub gemacht und es war immer sehr schön, aber niemals habe ich auch nur einen Augenblick lang mit dem Gedanken gespielt, wie es wäre, wenn das meine neue Heimat würde.

Manchmal allerdings werde ich von Leuten in Wien dazu gebracht, mir doch solche Fragen zu stellen.

[3: Beginn Episode mit Mann aus Gasthaus]

An ein Mal erinnere ich mich sehr gut. Ich war ungefähr zwölf Jahre alt. Da lief ich eines Tages unsere Gasse hinunter und stieß beinahe mit einem Mann zusammen, der aus einem Gasthaus auf den Gehsteig gestolpert kam. Ich konnte gerade noch abbremsen.

Er schwankte beträchtlich und ruderte mit den Armen, um die Balance wiederzuerlangen. Er hatte wohl, wie schon etliche vor ihm, die Stufe beim Ausgang übersehen. Ich jedenfalls hatte mit seinem Stolpern wirklich ganz und gar nichts zu tun, trotzdem packte ihn bei meinem Anblick die Wut. Er verstellte mir, kaum dass er wieder aufrecht stand, den Weg, stieß mich am Arm und blies mir seinen Alkoholatem entgegen, dass mir übel wurde:

„Schleich di!"Hasserfüllt sah er mich an und wollte mich noch einmal stoßen. "Schleich di!“, wiederholte er sich. „Geh hin, wo'st herkummst. Mir brauchen eich do net. Host g'heat?"

Da schlich ich mich in der Tat sehr schnell und ging dorthin, wo ich herkam ein paar Meter weiter zu unserer Wohnung, dorthin, wo mein Zuhause ist, wo ich immer gelebt habe, seit ich auf der Welt bin.

Ich war sehr erschrocken. Wenn es nicht heller Tag gewesen wäre mit vielen Leuten auf der Straße, und wenn dieser Typ sicherer auf den Beinen gewesen wäre, dann hätte er mich vielleicht nicht so ohne Weiteres abziehen lassen.

Vergessen konnte ich diese Begegnung bis heute nicht.

[3: Ende Episode mit Mann aus Gasthaus]

Für mich war immer klar, wo meine Heimat ist. - Wien! Ich liebe Wien! 
Aber dieser Vorfall hat mir klargemacht, dass ich für einige Wiener nicht hierher gehöre.

Nicht dass ich denen irgendetwas getan hätte. Wirklich nicht.

Es ist auch nicht so, dass ich nicht genug Deutsch könnte. Ganz und gar nicht. In meiner Klasse war ich immer die Beste in Deutsch. Es ist einfach deswegen, weil mein Haar nicht vom Frisör, sondern von Natur aus gelockt ist, und meine Haut nicht im Solarium braun geröstet, sondern deshalb getönt ist, weil Papa dunkelhäutig ist.

Einen anderen Grund kann es ja nicht geben, dass mich jemand, der mich gar nicht kennt, einfach wegjagen möchte.

Von Natur aus Locken und dunklerer Teint: Raus!

Die allermeisten Leute versichern mir aber, wenn man irgendwie darauf zu sprechen kommt - mitunter auch, wenn es gar nicht zum Thema passt, die Hautfarbe eines Menschen sei ihnen egal. Und überhaupt, sie hätten nichts gegen Ausländer.

Ich bin kein Ausländer. Auch keine Ausländerin. Aber schon okay. Ich schau halt so aus. Macht nichts. Ich bin froh, wenn sie nichts gegen mich haben.

Komische Sachen erlebe ich aber schon oft - obwohl man nichts gegen mich oder gegen Papa hat.

Das fällt mir seit dem Vorfall im Sandkasten immer wieder auf.

Das Seltsame dabei ist, dass genau die, die gerne betonen, dass sie nichts gegen Ausländer haben, sich oft so verhalten, dass ich das Gefühl bekomme, Ausländer und alle, die so ausschauen oder dem Namen nach welche sind, in Wirklichkeit doch ein ziemliches Problem für sie darstellen.

Anfangs habe ich mich ja immer mehr oder weniger gefreut, wenn jemand so besonders nett zu uns war. Toll! Soo lieb! Die haben nichts gegen uns, obwohl obwohl wir doch -eindeutig anders sind.

[4: Krankenhausepisode]

Ich war in der zweiten Klasse Volksschule, da musste Mama einmal zu einer Operation ins Krankenhaus. Dort angekommen, ging Mama schon voraus auf ihr Zimmer, während Papa und ich noch etwas zu erledigen hatten. Als wir ein wenig später das Krankenzimmer betraten, war Mama in einem, wie mir schien, freundlichen Gespräch mit ihren zwei Bettnachbarinnen und ich freute mich, dass sie nette Frauen im Zimmer hatte. Aber als die beiden uns erblickten, verstummten sie abrupt und starrten uns fast entsetzt an.

Mama stellte uns vor: „Meine Tochter. Mein Mann.“

Sie begrüßten uns verlegen und wirkten äußerst peinlich berührt. Anschließend nestelten sie in ihren Sachen herum und Mama sagte leise zu uns: „Yala!“

In der Halle unten erklärte sie uns die etwas seltsame Szene.

Sie sei ins Zimmer gekommen, da habe sie die Frauen in heller Aufregung miteinander diskutierend vorgefunden. Mama hätte sie freundlich begrüßt und sich als neue Bettnachbarin vorgestellt.

„SIE sind das!?", hätte eine ausgerufen.

„Dem Himmel sei Dank“, hätte sie die Hände zur Zimmerdecke erhoben. 
Die andere hätte zustimmend aufgeatmet und erklärt:

„Wir haben geglaubt, die tun uns eine Ausländerin rein, müssen Sie wissen. Zum Glück haben sie das geändert. Da ist nämlich ein ausländischer Namen an Ihrem Bett und vor der Tür.“

„Das werden sie schon richtigstellen“, hätten sie Mama beruhigt. „Sagen Sie's der Schwester! Ich kann sie anläuten.“

„Na, jetzt bin ich aber froh! Na wirklich!“Die Erste hätte an ihr Herz gegriffen, als wäre sie knapp einem Herzinfarkt entgangen.

„Man ist ja heute nirgends mehr vor denen sicher. Die sind ja überall und am liebsten legen sie sich ins Krankenhaus. Kein Wunder, dass man da sensibilisiert ist."

In dem Moment waren Papa und ich auf der Bildfläche erschienen.

Mama musste eine gute Woche im Krankenhaus bleiben, und wenn es irgendwie möglich war, gingen wir bei unseren Besuchen immer in die Halle.

Das hatte zwei Gründe: Erstens war es im Zimmer immer so laut, dass man sich nicht richtig unterhalten konnte. Denn die zwei Frauen, wenn sie gerade einmal keinen Besuch hatten, telefonierten stundenlang. Lautstark, damit nur niemandem ein Wort entging, schilderten sie allen ihren Angehörigen der Reihe nach in den jeweils fast gleichen dramatischen Formulierungen das Neueste vom Tag. Wie eine Krankenhaus-Soap per Telefon, die zehnmal am Tag wiederholt wird. Von A, wie Aufwachen bis zu Z, wie Zubettgehen. - Hahaha, Witzchen, man sei ja im Bett. Und weil beide meist gleichzeitig telefonierten, mussten sie sich gegenseitig überschreien.

Es gab aber auch Pausen dazwischen. Und was in denen geschah, das war der zweite Grund, der uns in die Halle flüchten ließ, so oft dies nur möglich war.

In diesen Pausen übertrafen sie sich gegenseitig an Freundlichkeit uns und besonders mir gegenüber. Sie fanden mich so was von herzig und lieb und intelligent und süß und wie ich schön reden täte und so weiter. Einfach allerliebst.

„Und so ein gutes Benehmen, nein wirklich! Da können sich die Unsrigen was abschauen." Ich war keine Ihrige.

Mama erklärten sie neben mir: „Gerade die Mischlingskinder sind ja oft besonders hübsch.“

Wenn Mama mit den Frauen allein war, wurden sie nicht müde, ihr zu versichern, dass sie „absolut nichts gegen Ausländer“ hätten. Es sei ihnen richtig peinlich, wie das am Anfang gelaufen sei. Mama hätte sie da sicher missverstanden. Da sei wahrscheinlich irgendwas falsch rübergekommen.

Verwunderliche Feststellung, denn Mama hat mit ihnen gar nie darüber geredet, was sie wie verstanden hat.

Es sei halt nur einfach so, erklärten sie ihr, man sei ja nicht zum Vergnügen im Krankenhaus und die Ausländer hätten bekanntlich immer so viel Besuch. Die hätten eben so viele Verwandte, das sei einfach so. Schön und gut. Aber in einem Krankenzimmer sei das eine Zumutung. Da würde der Mensch halt seine Ruhe brauchen.

Das mit der Ruhe sah Mama auch so und sie unterschrieb schließlich einen 
Revers, dass sie früher nach Hause gehen konnte, weil sie wirklich Ruhe brauchte.

Die beiden Frauen aber habe ich in Erinnerung behalten. Ihre Freundlichkeiten mir gegenüber, wenn sie mich so überschwänglich lobten, obwohl sie ja gar nichts von mir wussten, und wenn sie mir von den Süßigkeiten in ihren Nachttischen etwas anboten, die waren mir mehr als unangenehm. Sie hatten sich so aufgeregt, nur weil sie einen ,ausländischen“ Namen gelesen hatten.

[5: Tiergartenepisode]

Auf einer kleinen Wanderung durch den Lainzer Tiergarten sind wir auch wieder einer Frau begegnet, die absolut nichts gegen Ausländer hatte. Im Gegenteil.

Als wir, Mama, Papa und ich, uns zum Picknick an einem dieser Holztische niederließen, die überall auf Rastplätzen aufgestellt sind, und den Inhalt unserer Rucksäcke auf dem Tisch ausbreiteten, äugten viele Wanderer im Vorbeigehen neugierig zu uns her.

Wenn jemand isst, das nur am Rande, weil es mir so oft schon aufgefallen ist, ist das für die meisten Menschen sehr interessant. Im Tiergarten sind bei den einzelnen Gehegen deshalb ja auch eigens die Fütterungszeiten angegeben.

Na egal, an dem schönen Tag schauten alle freundlich und einige wünschten uns sogar guten Appetit.

Eine ältere Frau blieb, die Hände am Rücken, das Bäuchlein vorgestreckt, am Wegrand stehen und fragte, was es denn Feines gebe.

Mein Vater wäre kein Ägypter, wenn er da nicht sofort eine Einladung ausgesprochen hätte. Und seinen Einladungen entkommt man kaum, aber meist will das auch gar niemand.

Diese Frau jedenfalls schien geradezu darauf gewartet zu haben.

Im nächsten Augenblick saß sie bei uns am Tisch und langte beherzt zu.

Sie ließ sich, hoch interessiert, alle Speisen erklären und wie sie zubereitet würden.

Die gegrillten Melanzani mundeten ihr so gut, dass fix wie nix alle in ihrem Mund verschwanden und das, obwohl die Frau gleichzeitig pausenlos redete.

Von den Falafeln hingegen fühlte sie sich betrogen. Was so aussieht wie ein „Fleischlaberl“, habe auch eines zu sein.

Ägypten, sagte sie, das sei doch das mit den Pyramiden. Da würde man ja bis heute noch nicht wissen, wie die die „Steiner" aufeinandergetürmt hätten. Aber früher seien die Menschen ja überhaupt gescheiter gewesen.

Die Verblödung sei erst mit dem Fernsehen und dem Internet gekommen. Und in der Schule - da täte man schon überhaupt nichts mehr lernen. Das sei ja auch gar nicht möglich „bei den ganzen Ausländern“. (Wohl ein Glück, dass die halben nicht auch noch in der Schule sind... !)

Da stockte sie, warf uns einen kurzen prüfenden Blick zu und versicherte dann, dass sie „absolut nichts gegen Ausländer“ hätte. Wir sollten das bloß nicht missverstehen!

„Meine Schwester in Graz, die hat einen Nachbarn, der ist auch ein Pakistani, und der ist so was von hilfsbereit, das ist einmalig", schwärmte sie. 
„Vom Einkaufen bis Kohlen schleppen - in den vierten Stock rauf! - Der macht alles. Also wirklich, da kann man nichts sagen."

Ohne den würde die Schwester "glatt" eine Heimhilfe brauchen. Und dem müsse sie nichts zahlen.

„Diese Leute haben nämlich einen unwahrscheinlichen Stolz“, erklärte sie uns.

Die Schwester hätte ihm mal einen warmen, „tadellosen“ Wintermantel von ihrem verstorbenen Mann angeboten, aber:

„Nix da! Sie wissen das ja“, sagte sie zu Papa, „Sie täten sicher auch nichts nehmen. Also nichts gegen die Pakistani!“

„Ja“, sagte Papa, ,aber ich bin Ägypter.“

„Die da unten“, überging sie Papas Einwand, „haben einfach eine ganz eine andere Mentalität.“

Nach dieser Feststellung strahlte sie Papa gutmütig an und plötzlich lege sie ihr Köpfchen schräg und verengte prüfend die Augen.

„Also!“, platzte sie heraus, denn sie hatte eine Entdeckung gemacht. „Sie schauen dem Pakistani von meiner Schwester direkt ähnlich. Ja, wirklich!“”

Das sei geradezu verblüffend, wie ähnlich, nur sei Papa viel größer.

„Vielleicht kennen Sie ihn ja zufällig. Nadsch oder Nadschi oder so irgendwie heißt er. Ich werde meine Schwester fragen. Mein Nadsch, sagt sie immer, oder so irgendwas."

Papa musste sie enttäuschen:

„Ich kenne keinen Pakistani in Graz. Aber ich kenne eine Frau, die ist auch Finnin. Rossa oder Rosso oder so ähnlich heißt sie. Vielleicht kennen Sie die zufällig.“

Die Frau sah meinen Papa an, als ob er nicht ganz dicht wäre.

Dann fand sie, dass es Zeit für sie sei, sich wieder auf den Weg zu machen.

Als sie davon stapfte, sagte Mama: „Die denkt sich jetzt sicher, dass es auch blöde Pakistani gibt.“

Papas Witz mit der Finnin fand ich sehr passend. Ich meine, von Kairo nach Islamabad ist es mehr als siebenmal so weit wie von Wien nach Helsinki. 
Mahdy, Amina (2013): „In Between“ - Mein Leben in zwei Kulturen. In: Stippinger, Christa (Hrsg.): anthologie: preistexte 13. Wien: edition exil, 138-141

Amina Mahdy im Gespräch: „Beide Seiten haben für mich verständliche Argumente, und doch reden sie aneinander vorbei.“

Mein Vater ist in Ägypten geboren und vor etwa zwanzig Jahren nach Österreich gekommen, wo er meine Mutter kennen gelernt hat. Ich bin hier geboren und war immer nur urlaubsweise in Ägypten. Mit meinem Vater spreche ich nur Arabisch, weil meine Eltern unbedingt wollten, dass ich zweisprachig aufwachse, was ich als Vorteil sehe. Ich liebe Sprachen. In der Schule lerne ich Latein, okay, das spricht man nicht mehr, aber es hilft mir beim Verständnis anderer Sprachen, bei Englisch, Französisch und Spanisch, meinen anderen Schulsprachen, und ich möchte noch viele mehr lernen.

Meine Verwandten leben fast alle in Ägypten. Es sind so viele, dass ich was zum Schreiben brauche, um sie zu zählen. Besucht haben wir sie schon ein paar Jahre nicht mehr. Wir hoffen darauf, dass sich die politische Situation verbessert. Ich halte aber natürlich mit ihnen Kontakt über das Internet - auf Englisch, weil ich die arabische Schrift leider nicht ausreichend beherrsche.

Wenn ich in Ägypten bin, fühle ich mich als Ausländerin, obwohl es doch die Heimat meines Vaters und deshalb in gewisser Weise auch meine Heimat ist. Und von den Menschen dort werde ich als Touristin angesehen. Zu Hause fühle ich mich hier. Meine Heimat ist Österreich, und Deutsch empfinde ich als meine Muttersprache, in der ich mich auch viel besser ausdrücken kann.

Ich bin jetzt in der achten Klasse, stehe also kurz vor der Matura und freue mich schon darauf, dass ich die Schule bald hinter mir haben werde. Ich gehe zwar gerne zur Schule, aber schön langsam reicht es. Danach werde ich versuchen, diesen Mördertest fürs Medizinstudium zu schaffen, oder, falls mir das nicht gelingt, Englisch studieren. Im Moment habe ich daher wenig Zeit fürs Schreiben. Trotzdem glaube ich, dass dies etwas ist, das ich immer tun werde. Beim Schreiben kann ich meine Gedanken ordnen, Momente festhalten, Ereignisse objektivieren oder einfach meiner Fantasie freien Lauf lassen.

Ich habe schon als kleines Kind gesagt, dass ich Autorin werden will. Zwar habe ich nicht gewusst, was genau man da macht, aber ich habe immer gerne und viel gelesen, und jedes Mal, wenn ich mit einem guten Buch fertig war, habe ich mir vorgenommen, auch mal so etwas zu schreiben. Ich lese eigentlich jedes Genre und quer durch die Epochen. Früher habe ich nur auf Deutsch gelesen, derzeit überwiegend auf Englisch. Ich liebe diese Sprache. Seit kurzem schreibe ich auch persönliche Dinge auf Englisch.

Sehr gut schreiben kann ich auf Zugreisen. Da kann ich richtig abschalten. Ich bin dann nirgendwo richtig und fahre durch die Landschaft und das finde ich sehr inspirierend. Dafür habe ich immer meinen IPod bei mir. Da schreibe ich mir alles auf. Wenn ich zu Hause bin, schreibe ich nur in meinem Zimmer, denn wenn nebenbei jemand redet, lenkt mich das zu sehr ab. Im Zug höre ich 
beim Schreiben immer Musik, am liebsten Vertrautes oder Soundtracks.

Der Text „In Between“ ist über viele Jahre hinweg entstanden. Mir ist immer wieder aufgefallen, wie viele Missverständnisse es zwischen den Kulturen gibt, wie viele Vorurteile. Immer öfter wurde mir auch bewusst, dass ich Sachen erlebe, mitunter ganz skurrile, die meine gleichaltrigen österreichisch aussehenden Freunde nicht erleben. Irgendwann habe ich dann begonnen, mir erst einmal nur für mich selbst Notizen dazu zu machen. Ich schreibe generell vieles auf, das mir so durch den Kopf geht. Für diesen Text habe ich gezielt Alltagserlebnisse gesammelt, die in diesem Zusammenhang stehen. Das können amüsante Erlebnisse sein, wie dass mich jemand auf Englisch anspricht oder mich fragt, woher ich komme - mich, die ich hier geboren bin und nie woanders gelebt habe.

„Aus Wien!“

„Nein, nein, ursprünglich?“

Es kann aber auch passieren, dass mich jemand nicht sehr freundlich auffordert, gefälligst dorthin zurückzukehren, wo ich herkäme. Wo auch immer das sein mag...

Ich habe verstanden, dass mich die Gesellschaft nicht als einen Teil von sich anerkennt, nur weil ich mich dazugehörig fühle. Aber, wie gesagt, auch in Ägypten werde ich nicht als Einheimische betrachtet. Wozu macht mich das also? Wenn ich in beiden Gesellschaften nicht zu Hause bin - bin ich heimatlos? Nein, so fühle ich mich nicht. Ich sehe mich als Österreicherin.

In meinem Umfeld gibt es zum Glück viele Menschen, die mich nicht als fremd betrachten, sondern mich akzeptieren. Für sie ist das Ganze gar kein Thema. Ich bin, wie man so sagt, „integriert", habe die österreichische Lebensweise und die Art zu denken verinnerlicht und befasse mich mit Kultur und Politik dieses Landes. Ich bin damit groß geworden und bin stolz darauf.

Durch meinen Vater kenne und verstehe ich aber auch das Ägyptische. Verhaltensweisen, die den Leuten hier fremd sind, kann ich nachvollziehen. In unserem Familienalltag sah ich mich von klein auf immer wieder als Vermittlerin. Oft habe ich mir an den Kopf gegriffen, weil ich nicht glauben konnte, dass man andere Standpunkte einfach nicht akzeptieren kann. Beide Seiten haben für mich verständliche Argumente, und doch reden sie aneinander vorbei. Das klingt jetzt so, als wäre bei uns zu Hause die Hölle los, aber eigentlich funktioniert es gut, das Nebeneinander zweier so unterschiedlicher Kulturen.

Es kann funktionieren. Es muss nur eine gewisse Bereitschaft da sein, und der Mut, sich für andere Perspektiven zu öffnen.

Eines Tages hatte ich ein sehr treffendes Bild vor Augen: Ein Gegenstand, von zwei Seiten betrachtet, kann zwei unterschiedliche Bilder ergeben. Ist deswegen eines der beiden Bilder falsch? Natürlich nicht. Im Gegenteil. Beide Bilder zusammen ergeben ein vollständigeres Bild des Gegenstandes. Genauso verhält es sich mit Ansichten. Die Österreicher haben ihre Perspektive - die Zuwanderer eine andere. Wäre es nicht eine schöne Vision, beide Perspektiven zu einem erweiterten Horizont zusammenzufügen? Könnte ich einen Beitrag dazu leisten? 
Aus meinen Notizen wurden Kurzgeschichten. Einerseits behandeln sie Erlebnisse einer „Ausländerin“ in Österreich, andererseits stellen sie die beiden Kulturen einander gegenüber. Ob dies für irgendjemanden von Interesse ist keine Ahnung! Mir persönlich half das Schreiben sehr, um besser zu verstehen, welchen Platz ich in dieser Welt einnehme. Als ich die Ausschreibung für den exil-Literaturpreis sah, habe ich die Texte noch einmal bearbeitet, in der Hoffnung, dass sie auch für andere von Nutzen sein könnten. 